Operative Techniken

Oper Orthop Traumatol 2019 · 31:275-283 https://doi.org/10.1007/s00064-019-0615-7 Eingegangen: 28. September 2018

Überarbeitet: 24. Januar 2019

Angenommen: 4. März 2019

Online publiziert: 25. Juni 2019

() Der/die Autor(en) 2019

\section{Redaktion}

M. Mayer, München

Zeichner

R. Himmelhan, Mannheim

\section{Vorbemerkungen}

Die dorsale atlantoaxiale Stabilisierung in Goel-Harms-Technik [4] ist der Standard zur Versorgung atlantoaxialer Instabilitäten, sei es durch Tumor, Trauma oder Entzündung. Diese Technik hat in der Vergangenheit die Verschraubung nach Magerl [3] in Kombination mit einer Gallie-Fusion [2] als Goldstandard abgelöst, da sie einige Vorteile bietet. Hier ist an die kompliziertere Lagerung bei der MagerlVerschraubung in Bezug auf die Reposition der Halswirbelkörper HWK 1/2 sowie auf die Notwendigkeit alternativer Schraubentrajektorien in HWK 2 bei atypischem Verlauf der Arteria vertebralis zu denken. Doch auch bei der GoelHarms-Technik sieht man aufgrund intraoperativer Verletzungen des HarmsPlexus im Rahmen der HWK-1/2-Fusion immer wieder einen relevanten Blutverlust. Um den Harms-Plexus intraoperativ nicht zu verletzen und relevante Blutung zu vermeiden, bietet sich in einem Teil der Fälle bei ausreichend großem Pedikel eine alternative Schraubenplatzierung mit HWK-1-Pedikelschrauben an $[5,6]$. Ein weiterer Vorteil dieser modifizierten Technik ist die erhöhte biomechanische Stabilität, welche im Kadavermodell nachgewiesen werden konnte [1].

Lennart Viezens ${ }^{1}$ - Stephan Sehmisch' - Lukas Weiser' - Marc Dreimann ${ }^{2}$. Wolfgang Lehmann'

' Klinik für Unfallchirurgie, Orthopädie und Plastische Chirurgie, Universitätsmedizin Göttingen (Direktor: Prof. W. Lehmann), Göttingen, Deutschland

${ }^{2}$ Klinik und Poliklinik für Unfall-, Hand- und Wiederherstellungschirurgie, Spine Center,

Universitätsklinikum Hamburg Eppendorf (Direktor: Prof. K.-H. Frosch), Hamburg, Deutschland

\title{
Dorsale Stabilisation der Halswirbelkörper HWK1/HWK2 modifiziert nach Goel-Harms mit HWK-1-Pedikelschrauben
}

\section{Operationsprinzip und -ziel}

Durch die Verwendung von C1-Pedikelschrauben bei der atlantoaxialen Stabilisation wird das Blutungsrisiko durch die größere Entfernung zum Harms-Plexus bei gleichzeitiger Erhöhung der biomechanischen Stabilität reduziert.

\section{Vorteile}

- Geringere Gefahr der Blutung aus dem Harms-Plexus

- Geringeres Risiko einer Affektion von der Nervenwurzel C2

- Revision auf klassische Massa-lateralis-Schraube nach Harms möglich

\section{Nachteile}

- Gefahr der Verletzung der A. vertebralis

- Nur bei ausreichend großem HWK-1-Pedikel möglich

- Gefahr des Schraubenausbruchs

\section{Indikationen}

- Atlantoaxiale Instabilität bei Tumor, Trauma oder Entzündung

\section{Kontraindikationen}

- HWK-1-Pedikel <3,5 mm

- Atypischer Verlauf der A. vertebralis

\section{Patientenaufklärung}

- Allgemeine Operationsrisiken

- Verletzung der A. vertebralis mit Gefahr einer zerebralen Ischämie

- Verlängerung der Instrumentation mit Einschluss des C1/C0-Gelenks

- Pseudarthrose

- Postoperativ persistierende Nackenschmerzen

- Eingeschränkte Beweglichkeit

- Blickdeviation

\section{Operationsvorbereitungen}

- Präoperative Computertomographie (CT) und exakte Analyse

- Analyse des geplanten Schraubenverlaufs

- Ggf. Magnetresonanztomographie (MRT), ggf. Angio-CT bei unklarem Verlauf der A. vertebralis

- Präoperative Bestimmung der Pedikelweite von HWK 1

- Degeneration der kaudalen Segmente mitbeachten

- Rasur des Hinterhaupts

- Antibiotikaprophylaxe nach Hausstandard

\section{Instrumentarium}

- Zervikales Schrauben-Stab-System

- Mayfield-Klemme, alternativ Kopfschale 


\section{Anästhesie und Lagerung}

- Intubationsnarkose mit invasiver Druckmessung

- Anlegen der Mayfield-Klemme in Rückenlagerung

- Umlagern in Bauchlage und Eingehen der Concorde-Position

- Kontrolle auf ausreichend gute Darstellbarkeit im Röntgen nach abgeschlossener Lagerung

Oper Orthop Traumatol 2019-31:275-283 https://doi.org/10.1007/s00064-019-0615-7

(c) Der/die Autor(en) 2019

L. Viezens · S. Sehmisch · L. Weiser · M. Dreimann · W. Lehmann

Dorsale Stabilisation der Halswirbelkörper HWK1/HWK2 modifiziert nach Goel-Harms mit HWK-1-Pedikelschrauben

\section{Zusammenfassung}

Operationsziel. Stabilisation des atlantoaxialen Übergangs durch eine alternative Schraubenposition im Halswirbelkörper 1 (HWK 1).

Indikationen. Instabilitäten von

HWK 1/HWK 2 durch Entzündung,

Tumor oder Trauma.

Kontraindikationen. Vorhandensein sehr kleiner Pedikel von HWK 1. Varianten des Verlaufs der Arteria vertebralis.

Operationstechnik. Über den klassischen Mittellinienzugang zur oberen HWS erfolgt die modifizierte Instrumentation von HWK 1 mit Pedikelschrauben anstelle der klassischen Harms-Massa-lateralis-Schrauben bei unveränderter Instrumentation von HWK 2. Anschließend erfolgt - je nach Indikation - die dorsale Spondylodese durch Eröffnung der Laminae und Anlagerung von keramischem Knochenersatzmaterial.

Weiterbehandlung. Bei mobilen Patienten erfolgt die zusätzliche Ruhigstellung in der weichen Halskravatte für 6 Wochen; die volle
Belastbarkeit ist wieder nach 3-4 Monaten gegeben.

Ergebnisse. Im Zeitraum von Januar 2017 bis September 2018 erfolgten in der Klinik der Autoren 21 Stabilisationen des atlantoaxialen Übergangs. Das mittlere Alter betrug $72,52 \pm 15,45$ Jahre. Insgesamt wurden 42 Schrauben in HWK 1 gesetzt. Eine HWK-1Pedikelschraube konnte 21-mal (50\%) platziert werden. In den anderen 21 Fällen erfolgte die Instrumentation mit einer klassischen Harms-Massa-lateralis-Schraube. Komplikationen zeigten sich bei 3 Patienten. Insgesamt erscheint bei Beachtung der Kontraindikationen die Instrumentation von HWK 1 mit Pedikelschrauben als eine sichere Alternative zur Instrumentation mit HarmsSchrauben.

\section{Schlüsselwörter}

Wirbelsäule · Halswirbelkörper · Dens axis . Subaxiale Instabilität · Atlantoaxiales Gelenk

\section{Dorsal stabilization of C1/C2 modified according to Goel-Harms with $\mathrm{C} 1$ pedicle screws}

\section{Abstract}

Objective. Stabilization of the atlantoaxial transition by an alternative screw position in C1.

Indications. Instabilities $\mathrm{C} 1 / \mathrm{C} 2$ due to inflammation, tumor or trauma.

Contraindications. Presence of a very small pedicle of $\mathrm{C} 1$. Variations in the course of the vertebral arteries.

Surgical technique. The midline approach to the upper cervical spine is used for the modified instrumentation of $\mathrm{C} 1$ with pedicle screws instead of Harms screws and for the unaltered instrumentation of $\mathrm{C} 2$. Depending on the indication, dorsal spondylodesis is performed by opening the laminae and attaching ceramic bone substitute material. Postoperative management. In mobile patients, additional immobilisation with a soft collar is recommended for 6 weeks. Full recovery is given 3-4 months after surgery. Results. From January 2017 to September 2018, 21 stabilizations of the atlantoaxial transition were performed. The mean age was $72.52 \pm 15.45$ years. A total of 42 screws were placed in C1. In all, 21 (50\%) C1 pedicle screwscould be placed, and in other 21 cases Harms screws were used. Complications were seen in 3 patients. Overall, considering the contraindications, the instrumentation of $\mathrm{C} 1$ with pedicle screws appears as a safe alternative to instrumentation with Harms screws.

Keywords Spine - Cervical vertebra axis - Dens axis . Cervical instability · Atlanto axial joint 


\section{Operationstechnik}

(• Abb. 1, 2, 3, 4, 5, 6, 7, 8, 9, 10)

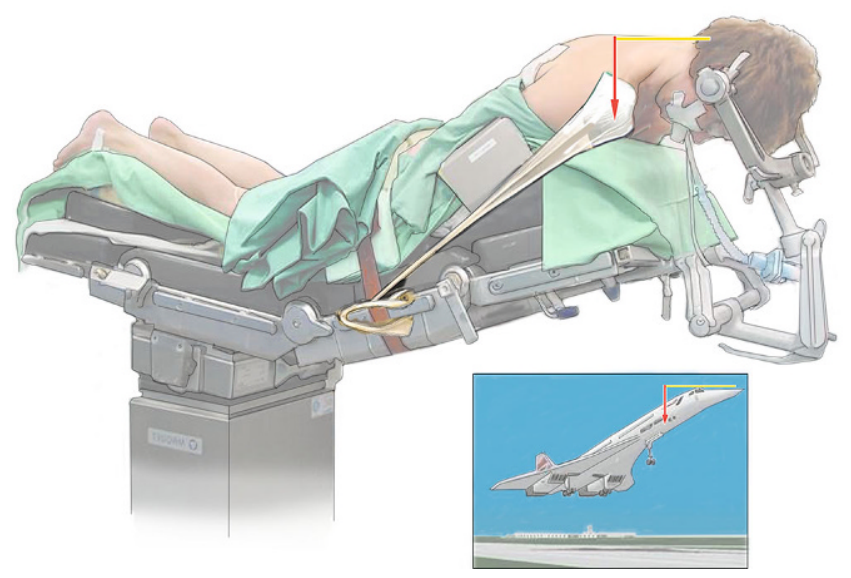

Abb. 1 ॥ Zunächst erfolgt die Lagerung des Patienten in Rückenlage. Das "Team time out" mit der Anästhesie wird nach Standard durchgeführt. Anschließend erfolgt die Anlage der Mayfield-Klemme $1 \mathrm{~cm}$ kranial der Ohren, die achsgerechte Umlagerung in die Bauchlage gemeinsam mit dem Team der Anästhesie und anschließend das Eingehen der Concorde-Position. Um den intraoperativen Blutverlust zu minimieren, sollte der Operationssitus oberhalb des Herzniveaus liegen. Hierbei ist insbesondere auf die Blickrichtung sowie die Blickachse des Patienten zu achten, um eine postoperative Blickdeviation zu vermeiden. Nun sollte, insbesondere bei vorliegenden Instabilitäten, ein zügiges Röntgenbild zur Verifikation einer guten Reposition atlantoaxial erfolgen. Anschließend erfolgt die Rasur des Nackens und des Hinterhaupts bis zur Hinterhauptsschuppe. Die Schultern werden mittels Pflasterverband nach kaudal ventral heruntergetapet. Anschließend erfolgt das Abwaschen und Abdecken von der Hinterhauptsschuppe bis zur mittleren Brustwirbelsäule

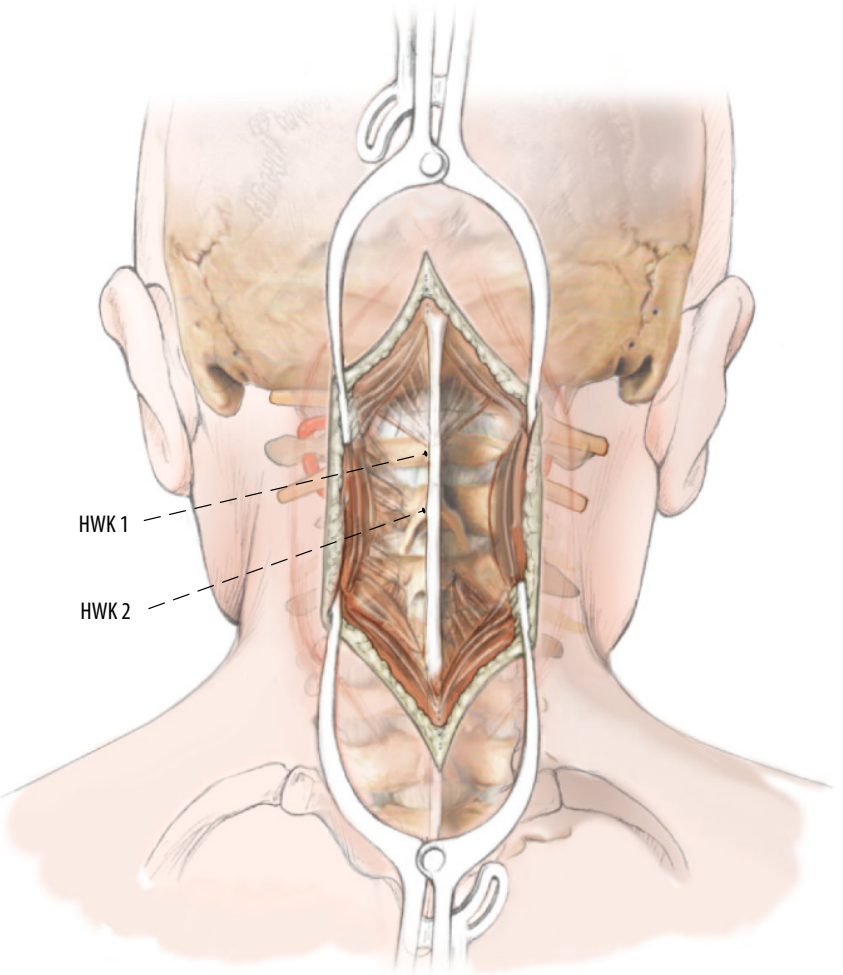

Abb. $2 \Delta$ Nun erfolgt der Hautschnitt vom Hinterhaupt bis zum Dornfortsatz von Halswirbelkörper HWK 7. Anschließend Einsetzen zweier oberflächlicher Wundsperrer und mit dem Monopolar Präparation bis auf den Processus spinosus von HWK 2. Anschließend subperiostale Ablösung der Nackenmuskulatur unter Zuhilfenahme eines Cobb-Raspartoriums und des Monopolar. Nach Darstellung des kranialen Rands der Lamina von HWK 2 erfolgt die Präparation von HWK 1. Hierzu wird zunächst das Tuberculum posterius ertastet und anschließend ebenfalls mit dem Monopolar die Muskulatur vom Atlasbogen abgelöst. Hierbei ist konsequenter Knochenkontakt mit dem Monopolar zu halten, da kranial die A. vertebralis und kaudal der Harms-Plexus Komplikationsgefahren bieten. Anschließend Abstopfen mit einer ausgezogenen Kompresse und Präparation der Gegenseite 


\section{Operative Techniken}

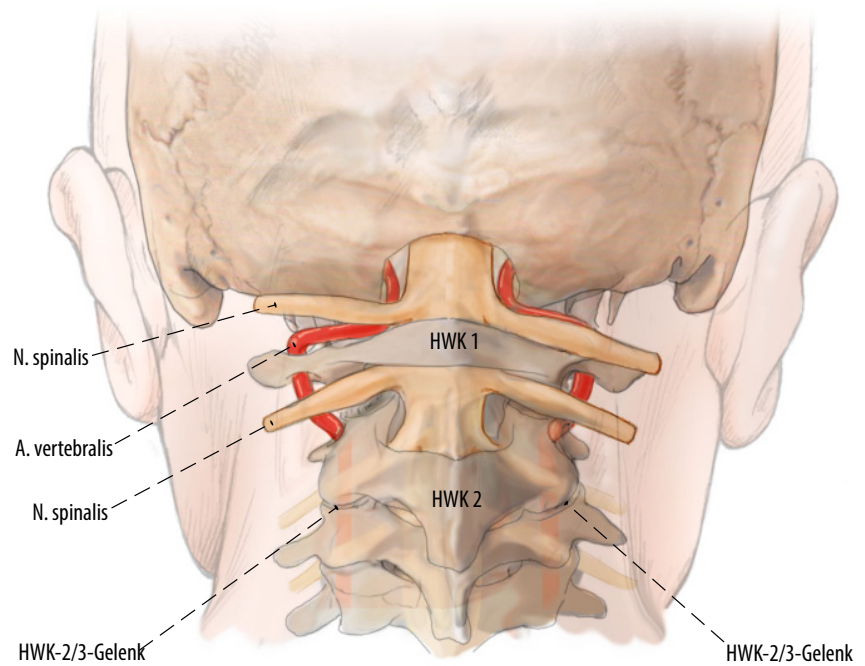

Abb. $3 \Delta$ Anschließend Entfernen der Kompressen und Tiefersetzen des Wundsperrers. Wichtig ist eine komplette knöcherne Darstellung der Wirbelbögen der Halswirbelkörper HWK 1 und HWK 2; das HWK-2/3-Gelenk sollte beidseits geschont worden sein. Die Nackenfaszie sollte bis zum Processus spinosus von HWK 6 inzidiert worden sein, um eine ausreichende Konvergenz für die HWK-2-Schrauben zu erlauben. Alternativ ist auch eine laterale perkutane Zusatzinzision möglich, um den medianen Zugang möglichst klein zu halten. Bluttrockenheit sollte vor den nächsten Schritten vorliegen
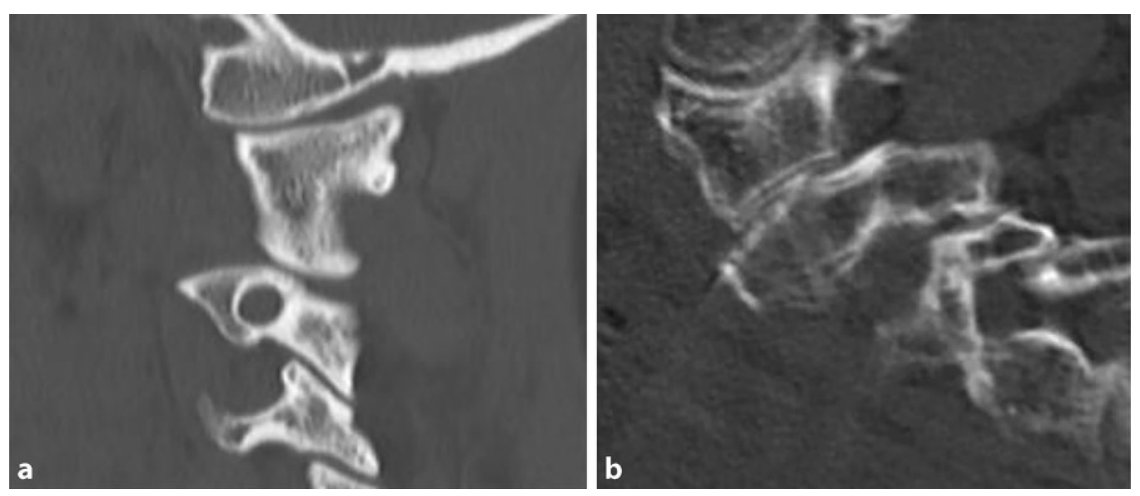

Abb. 4 \ Zunächst sollte nun, in Abhängigkeit der vorliegenden Anatomie, a high-riding A. vertebralis, b ausreichend großer, knöcherner Korridor für eine Pedikelschraube, die Instrumentation vom Halswirbelkörper HWK 2 entweder mit einerPedikelschraube oder mit einer Isthmusschraube erfolgen 


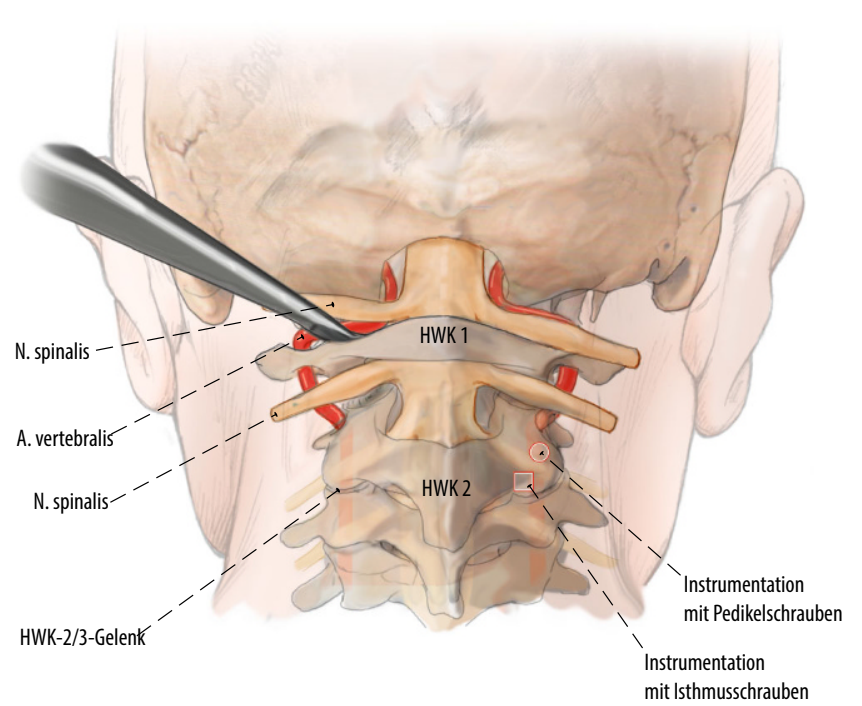

Abb. 5 A Für die Instrumentation sollte die Massa lateralis von Halswirbelkörper HWK 2 komplett dargestellt sein. Im Fall der Instrumentation mit Pedikelschrauben befindet sich der anatomische Eintrittspunkt im oberen lateralen Quadranten. Wenn eine Isthmusschraube gesetzt werden soll, befindet sich der Eintrittspunkt zentral in der kaudalen Hälfte der Massa lateralis. Anschließend wird unter fluoroskopischer Kontrolle oder mit Hilfe des Navigationssystems mit einem 2,4 mm dicken Bohrer vorgebohrt. Bei Erreichen der geplanten Tiefe erfolgt das Austasten des Schraubenlochs zum Ausschluss einer Perforation des Pedikels. Anschließend wird eine Vollgewindeschraube eingedreht. Nach dem beidseitigen Besetzen von HWK 2 erfolgt die Instrumentation von HWK 1

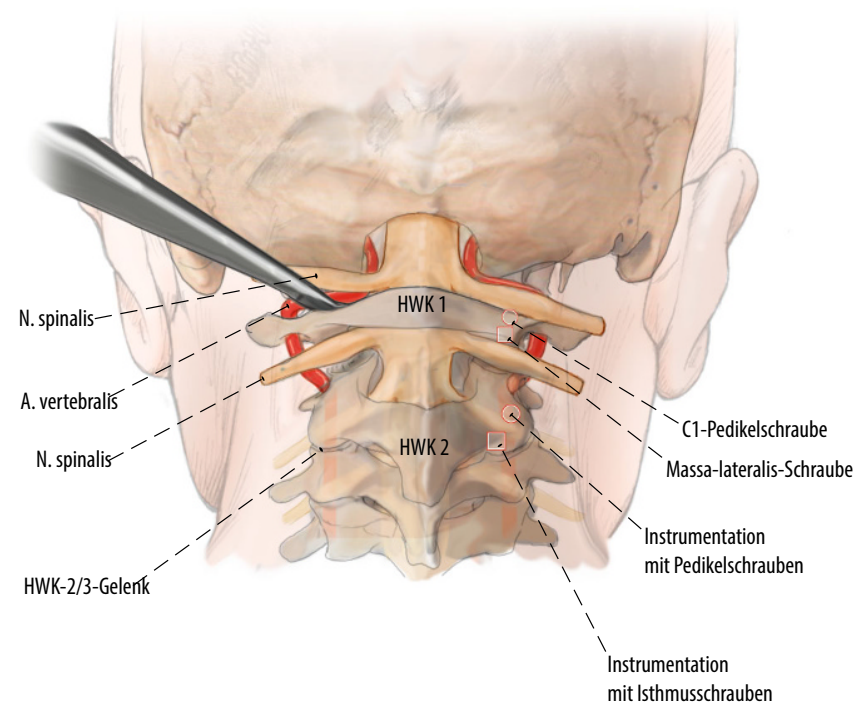

Abb. 6 ム Für die Instrumentation von HWK1 sollte im präoperativen Computertomogramm die Entfernung des Eintrittspunkts für eine HWK-1-Pedikelschraube vom Tuberculum posterius ausgemessen werden. Anschließend ertastet man mit dem Dissektor von kaudal die mediale Begrenzung des Pedikels von HWK 1. Mit einem zweiten Dissektor wird am Oberrand der Lamina von HWK 1 die A. vertebralis unterfahren; dieser Dissektor wird im Weiteren von dem Assistenten gehalten 


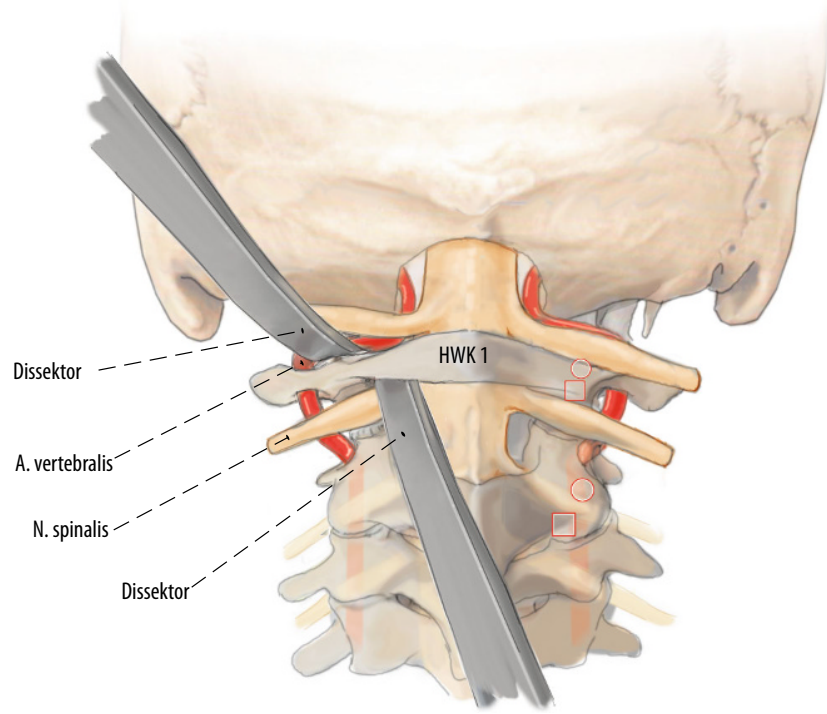

Abb. 7 A Anschließend wird mit der 1,8 mm großen Diamantfräse transpedikulär vorgebohrt, bis die Massa lateralis des Halswirbelkörpers HWK 1 erreicht wird. Hierbei ist auf eine geringe Konvergenz von ca. $10^{\circ} \mathrm{zu}$ achten. Eine Rotation des sehr mobilen HWK 1 durch den Druck der Fräse sollte hierbei berücksichtigt werden. Das Vorbohren sollte unter Kontrolle im seitlichen Röntgenbild oder mit Hilfe eines Navigationssystems erfolgen. Bei navigiertem Bohren sollte die Referenzklemme aufgrund der Mobilität des HWK 1 an den Wirbelbogen von HWK 1 fixiert werden. Alternativ ist eine Fixierung auch an dem Dorn von HWK 2 möglich, führt aber aufgrund der Mobilität atlantodental zu einer deutlich größeren Ungenauigkeit der Navigation. Anschließend kann nun erneut mit dem 2,4-mm-Bohrer in das eben geschaffene Loch eingegangen und bis zur ventralen Kortikalis von HWK 1 vorgebohrt werden. Anschließend sollte auch hier ein vorsichtiges Austasten erfolgen. Anschließend kann jetzt eine 3,5-mm-Vollgewindeschraube eingedreht werden. Eine bikortikale Schraubenlage ist zu tolerieren. Anschließend kann der vom Assistenten gehaltene Dissektor entfernt und die gegenseitige HWK-1-Schraube in gleicher Technik gesetzt werden. In der Erfahrung der Autoren bietet sich anschließend ein intraoperativer dreidimensionaler (3-D) Scan an, um eventuelle Schraubenfehlagen zu korrigieren und spätere Revisionen zu vermeiden. Hierbei ist dann jedoch die Anwendung einer Karbon-Mayfield-Klemme erforderlich

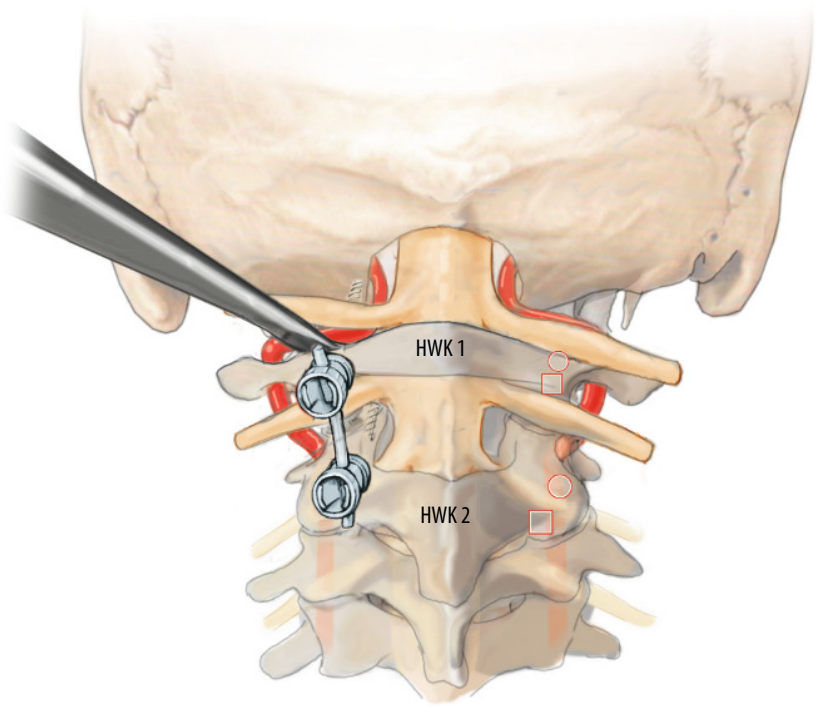

Abb. 8 ॥ Nach erfolgreichem Setzen allerSchrauben erfolgt das Einbringen der Längsverbinder. Hierbei ist insbesondere am kranialen Ende auf eine angemessene Stablänge zu achten, um ein Anschlagen des Hinterhaupts unbedingt zu vermeiden. $H W K$ Halswirbelkörper 


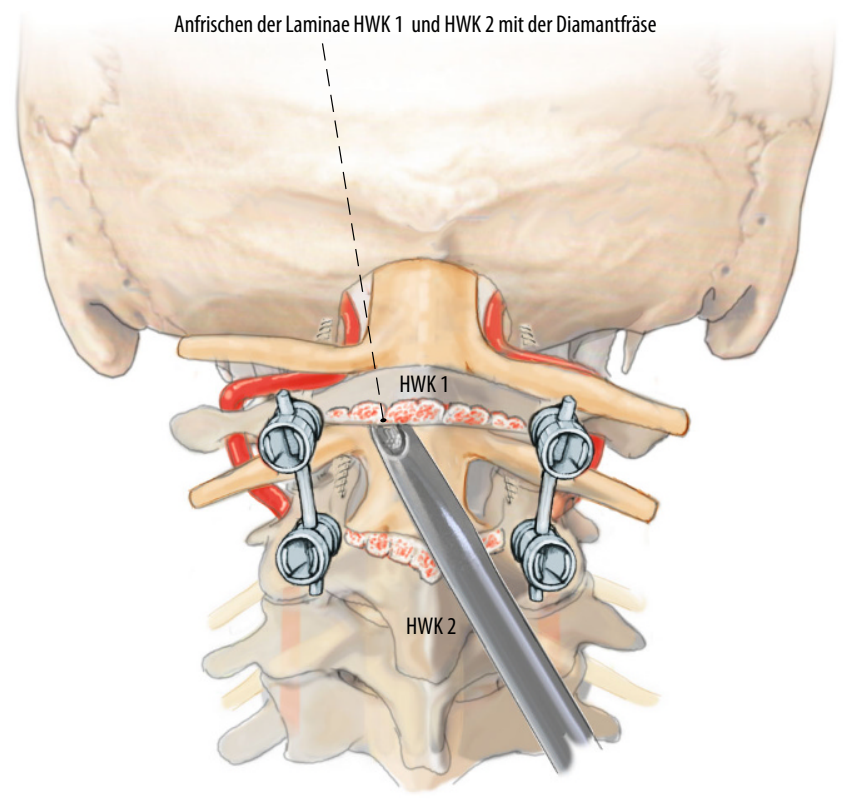

Abb. $9 \Delta$ Anschließend sollte je nach Indikation eine Spondylodese erfolgen. Hierzu muss der kaudale Anteil des Bogens von Halswirbelkörper HWK 1 dorsal und der kraniale Anteil der Lamina von HWK2 mit der Diamantfräse oder einer Stanze eröffnet und anschließend keramischer Knochenersatz oder autologe Spongiosa angelagert werden. Anschließend abschließende Wundspülung, Einlage zweier 12-Charrière-Redondrainagen und sorgfältiger Wundverschluss. Eine Refixation der vom Dornfortsatz HWK 2 abgelösten Muskulatur ist unbedingt durchzuführen, um postoperativen Beschwerden des Patienten vorzubeugen. Hierbei wird zunächst die Nackenfaszie wieder an die Dornfortsätze adaptiert. Anschließend erfolgt die wasserdichte fortlaufende Fasziennaht mit Vicrylfaden der Stärke 2. Anschließend Subkutannaht und Hautnaht in Donati-Rückstichtechnik. Nach dem sterilen Verband Umlagerung in Rückenlage und Entfernen der Mayfield-Klemme. Erst jetzt werden die Redondrainagen geöffnet und eine weiche Halskravatte angelegt. Sollte es zu Nachblutungen aus den Läsionen der Mayfield-Klemme kommen, kann ein tief gestochener Einzelknopf die Blutung stillen. Das postoperative Röntgenbild erfolgt am 2. postoperativen Tag
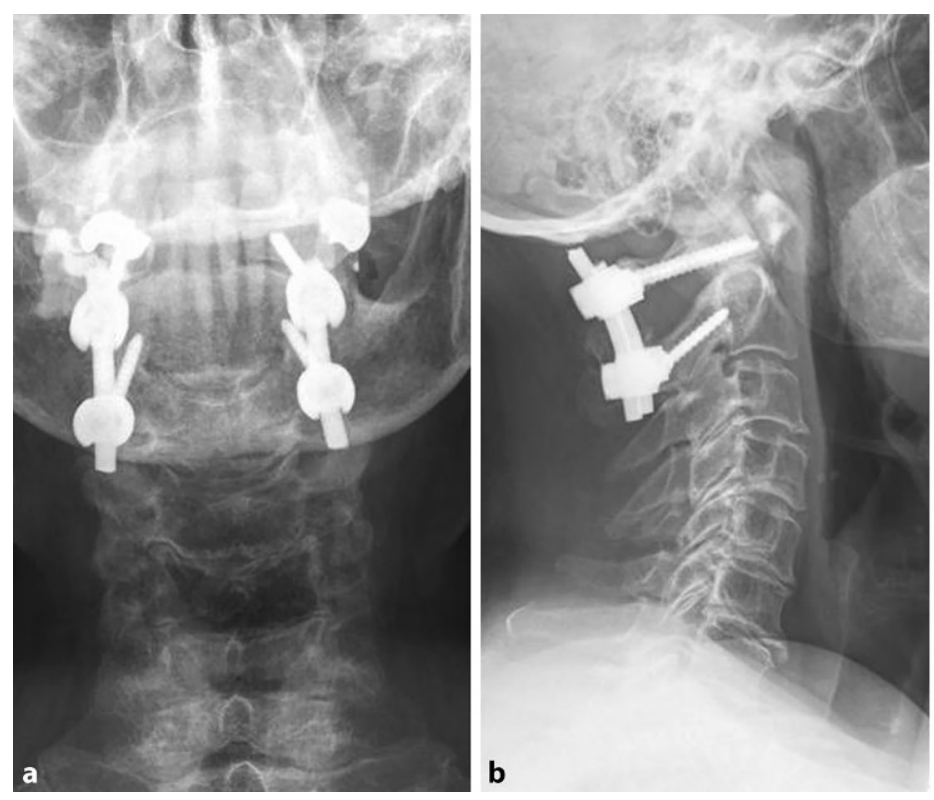

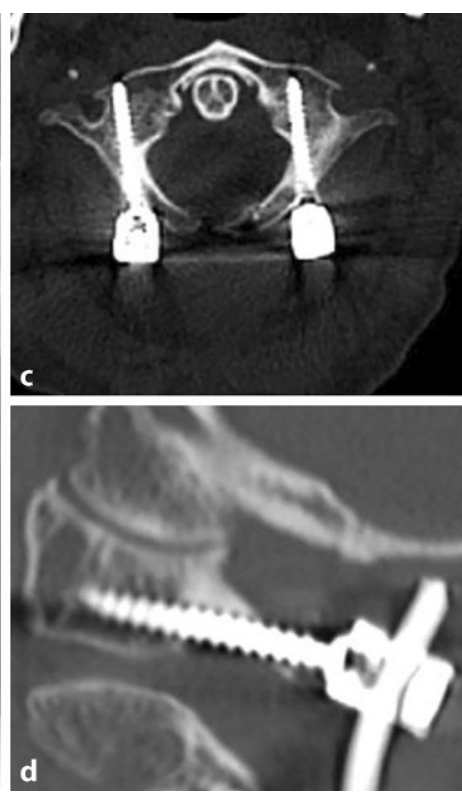

Abb. $10<$ Nativröntgenbild a.-p. (a) und seitlich (b) einer modifizierten Goel-Harmsverschraubung mit Pedikelschrauben in Halswirbelkörper HWK 1. Auf dem axialen (c) und sagittalen (d) Computertomographieschnitt ist die genaue Lage der Pedikelschraube zu erkennen 


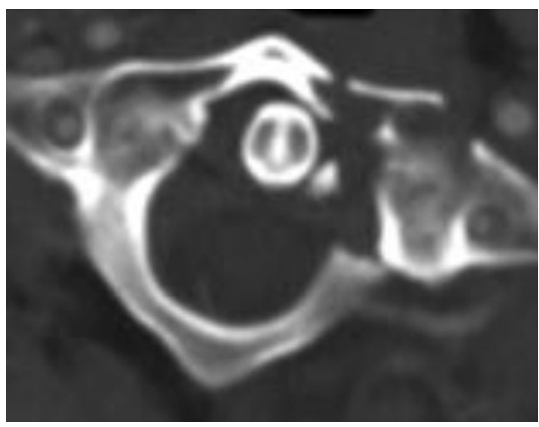

Abb. 11 A Frakturverlauf durch den Eintrittspunkt für die Pedikelschraube an Halswirbelkörper HWK 1

\section{Besonderheiten}

Beim Vorliegen von Atlasfrakturen kann es im Bereich der Fraktur schwierig sein, den Eintrittspunkt sicher darzustellen, ohne die A. vertebralis zu gefährden, so dass wir hier empfehlen, die klassische HWK-1-Massa-lateralis-Schraube zu platzieren ( $\bullet$ Abb. 11). Dies ist ebenso der Fall, wenn der Pedikel von HWK 1 $<3,5 \mathrm{~mm}$ misst.

\section{Postoperative Behandlung}

- Weiche Halskravatte für 6 Wochen bei mobilen Patienten

- Redondrainage für $48 \mathrm{~h}$ belassen

- Röntgenbild der Halswirbelsäule (HWS) in 2 Ebenen am 2. postoperativen Tag

- CT, wenn intraoperativ kein 3D-Scan durchgeführt wurde (• Abb. 12)

- Kein Heben oder Abstützen mit den Armen für 6 Wochen, um eine Faszieninsuffizienz zu vermeiden

- Entlassung nach 5-10 Tagen

- Röntgenkontrollen nach 6 Wochen, 6 Monaten und einem Jahr

- Ab 3 Monaten postoperativ ist die volle Belastungsfähigkeit gegeben

\section{Fehler, Gefahren, Komplikationen}

- Verletzung der A. vertebralis: Lokale Blutstillung; anschließend Angiographie, ggf. interventionelle Versorgung mit Stenting

- Wundinfekt: Bei persistierender Wundsekretion Revision mit Entnahme mikrobiologischer Proben

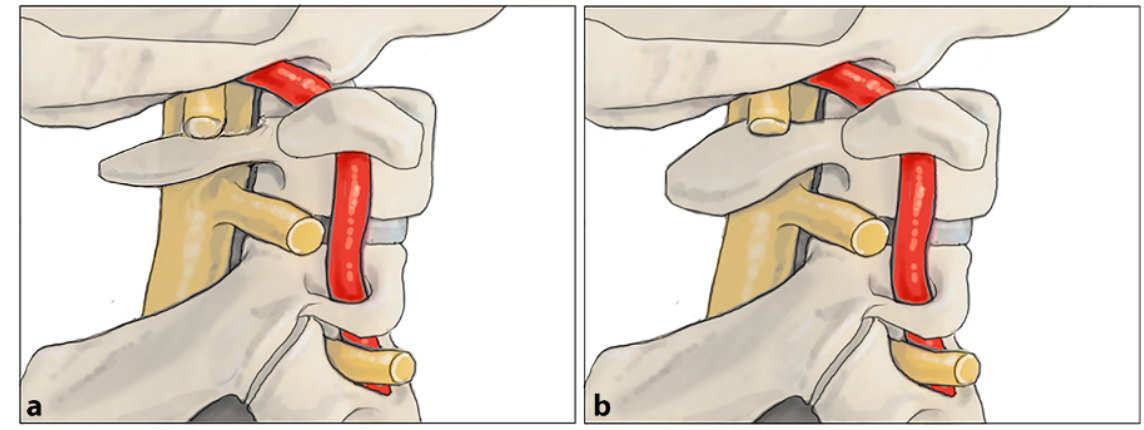

Abb. 12 \ Sehr schlanker Pedikel (a) von Halswirbelkörper HWK 1 versus ausreichend großer Pedikel (b)

und radikalem Débridement durch erfahrenen Operateur; kalkulierte antibiotische Therapie. Bei Keimnachweis antibiotische Therapie für 4 Wochen

- Verletzung der A. temporalis durch Mayfield-Klemme: Umstechung mit Hautnaht nach entfernen der Klemme

- Intraoperatives Ausbrechen der HWK-1-Schrauben: Verlängerung auf das Okkziput (präoperative Aufklärung!)

- Intraoperatives Ausbrechen der HWK-2-Schrauben: Alternative Schraubentechniken (Laminaschrauben), Verlängerung nach kaudal

\section{Ergebnisse}

Um Blutungskomplikationen aus dem Harms-Plexus zu vermeiden und eine erhöhte biomechanische Festigkeit der Schrauben $\mathrm{zu}$ erreichen, werden in unserer Klinik - wenn möglich HWK-1-Pedikelschrauben gesetzt. Um zu schauen, mit welcher Häufigkeit die Instrumentation von HWK 1 mit Pedikelschrauben möglich ist, haben wir einen 20-monatigen Zeitraum von Januar 2017 bis September 2018 in unserer Klinik ausgewertet. Dabei konnten 21 Patienten identifiziert werden, die aufgrund einer posttraumatischen Instabilität von HWK 1/2 mittels dorsaler Instrumentation behandelt wurden. Das mittlere Alter betrug 72,52 \pm 15,45 Jahre; 12 Patienten waren männlich. Insgesamt wurden 42 Schrauben in HWK 1 gesetzt, 21-mal (50\%) konnte eine HWK-1Pedikelschraube platziert werden. Bei
11 Patienten erfolgte eine beidseitige Instrumentation von $\mathrm{HWK} 1 \mathrm{mit} \mathrm{Pe}$ dikelschrauben und bei 9 Patienten die Instrumentation von HWK 1 beidseits mit klassischen Harms-Schrauben. In 3 Fällen war eine unterschiedliche Instrumentation nötig (rechts Pedikelschraube, links Harms-Schraube), da das Platzieren einer Pedikelschraube aufgrund des Frakturverlaufs durch den Eintrittspunkt (2-mal) oder eines intraoperativen Ausbruchs nach kaudal (1mal) nicht möglich war. In der Gruppe mit beidseitigen Harms-Schrauben betrug die mittlere Operationsdauer $170 \pm 92 \mathrm{~min}$, in der Gruppe mit den beidseitigen Pedikelschrauben war diese etwas kürzer (136 $\pm 37 \mathrm{~min})$.

Komplikationen zeigten sich bei drei Patienten: eine Verletzung der A. vertebralis mit der Notwendigkeit zum interventionellen Verschluss und einer dreimaligen Revision zur Hämatomentlastung. Eine intraoperative Reanimation aufgrund eines neu aufgetretenen atrioventrikulären Blocks dritten Grades. Die dritte beobachtete Komplikation war die Schraubenlockerung in HWK 1 mit progredienten Nackenschmerzen und der Notwendigkeit zur Revision ein Jahr postoperativ. Alle drei beobachteten Komplikationen wurden in der Gruppe der Patienten mit Harms-Schrauben beobachtet.

Abschließend zeigt sich, dass nach unserer Erfahrung in der Hälfte aller Patienten, die eine Instrumentation von HWK 1 benötigten, das Platzieren von HWK-1-Pedikelschrauben sicher möglich war. Aufgrund des geringeren Blutungsrisikos aus dem Harms-Ple- 


\section{\begin{tabular}{|l|l}
\hline & Lesetipp \\
\hline
\end{tabular}}

xus und der erhöhten biomechanischen Festigkeit empfehlen wir, wenn möglich, die Instrumentation mit HWK-1Pedikelschrauben.

\section{Korrespondenzadresse}

\section{PD Dr. Lennart Viezens}

Klinik für Unfallchirurgie, Orthopädie und Plastische Chirurgie, Universitätsmedizin Göttingen (Direktor: Prof. W. Lehmann) Robert-Koch-Straße 40, 37075 Göttingen, Deutschland

lennart.viezens@med.uni-goettingen.de

\section{Einhaltung ethischer Richtlinien}

Interessenkonflikt. L. Viezens, S. Sehmisch, L. Weiser, M. Dreimann und W. Lehmann geben an, dass kein Interessenkonflikt besteht.

Für diesen Beitrag wurden von den Autoren keine Studien an Menschen oder Tieren durchgeführt. Für die aufgeführten Studien gelten die jeweils dort angegebenen ethischen Richtlinien.

Open Access. Dieser Artikel wird unter der Creative Commons Namensnennung 4.0 International Lizenz (http://creativecommons.org/licenses/by/4.0/deed. de) veröffentlicht, welche die Nutzung, Vervielfältigung, Bearbeitung, Verbreitung und Wiedergabe in jeglichem Medium und Format erlaubt, sofern Sie den/die ursprünglichen Autor(en) und die Quelle ordnungsgemäßnennen, einen Linkzur Creative Commons Lizenz beifügen und angeben, ob Änderungen vorgenommen wurden.

\section{Literatur}

1. Fensky F, Kueny RA, Sellenschloh K et al (2014) Biomechanical advantage of $\mathrm{C} 1$ pedicle screws over C1 lateral mass screws: a cadaveric study. Eur Spine J 23:724-731. https://doi.org/10.1007/ s00586-013-3143-4

2. Gallie E Fractures and dislocations of the cervical spine. Am JSurg 5.https://doi.org/10.1016/S0002 9610(39)90309-0

3. Jeanneret B, Magerl F (1992) Primary posterior fusion $\mathrm{C} 1 / 2$ in Odontoid fractures: indications, technique, and results of transarticular screw fixation. J Spinal Disord 5:464-475

4. Kobbe P, Kandziora F, Hildebrand F, Scholz M (2017) Dorsale atlantoaxiale Stabilisierung in Goel-Harms-Technik. Unfallchirurg 120:701-706. https://doi.org/10.1007/s00113-017-0381-7

5. Resnick DK (2002) C1-C2 pedicle screw fixation with rigid cantilever beam construct: case report and technical note. Neurosurgery 50(3). https:// doi.org/10.1097/00006123-200202000-00039

6. Thomas JA, Tredway T, Fessler RG, Sandhu FA (2010) An alternate method for placement of $\mathrm{C}-1$ screws: Clinical article. J Neurosurg 12:337-341. https:// doi.org/10.3171/2009.10.SPINE08541

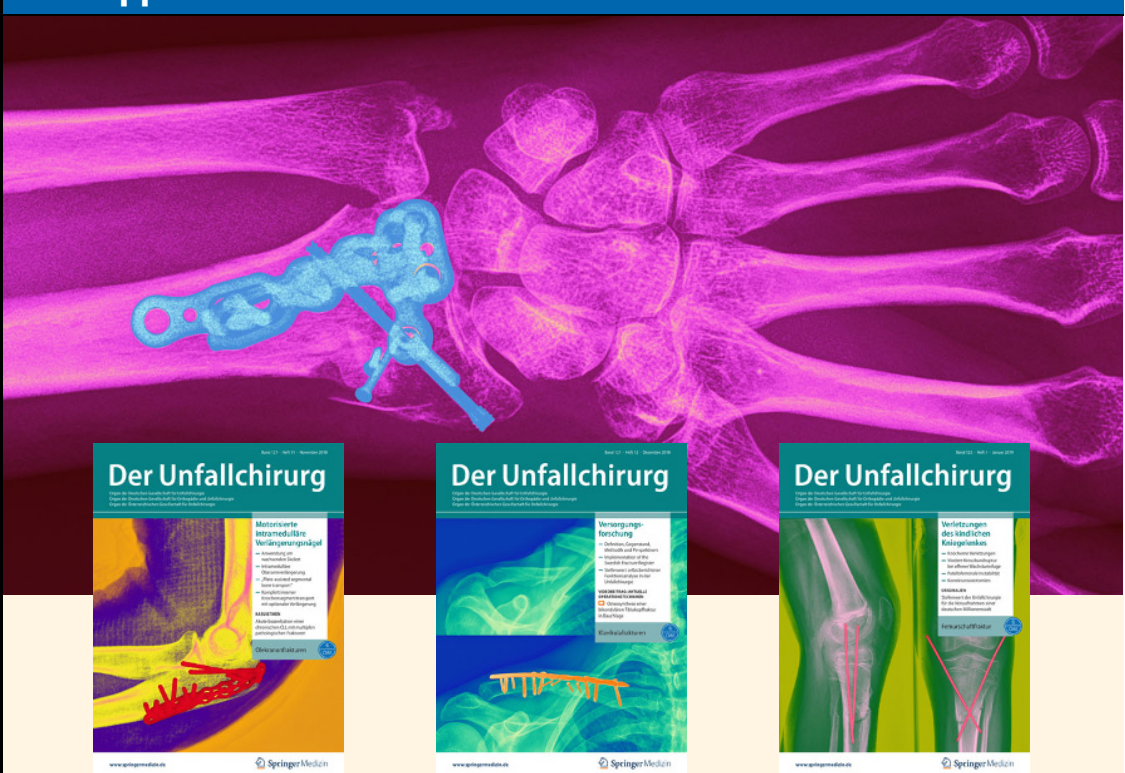

Der Unfallchirurg: Aktuelle Themenschwerpunkte

Der Unfallchirurg bietet Ihnen jeden Monat umfassende und aktuelle Beiträge zu interessanten Themen aus allen Bereichen der Unfall- und Wiederherstellungschirurgie.

Möchten Sie ein bereits erschienenes Heft nachbestellen?

Einzelne Ausgaben können Sie direkt bei unserem Kundenservice zum Preis von je

EUR 41,- zzgl. Versandkosten beziehen:

\section{Heft 4/2019: 3D-Druck}

- Aktueller Stand und neue Entwicklungen

- Planung, Druck und Aufbereitung

- Surgical applications of three-dimensional printing in the pelvis and acetabulum: from models and tools to implants

- 3D-Druck-Template-gestützte Reposition von Frakturen der langen Röhrenknochen

- CME: Mittel- und Vorfußfrakturen

\section{Heft 5/2019: Ellenbogenverletzungen im Kindesalter}

- Postoperativer Ulnarisschaden nach Kirschner-Draht-Osteosynthese der suprakondylären Humerusfraktur

- Therapieprinzipien und Outcome nach Frakturen des Condylus radialis im Wachstumsalter

- Korrekturosteotomien

- Ellenbogenluxationen

- Kasuistik: Blasse und pulslose Hand nach Luxation des linken Ellenbogens bei einem 9-jährigen Jungen

- CME: Perkutane Schraubentechniken an Beckenring und Acetabulum

So erreichen Sie unseren Kundenservice:

Springer Customer Service Center GmbH

Kundenservice Zeitschriften

Haberstr. 7, 69126 Heidelberg

Tel.: +49 6221 345-4303, Fax: +49 6221 345-4229

E-Mail: leserservice@springer.com

www.DerUnfallchirurg.de 